\title{
Safety and efficiency of emergency department assessment of chest discomfort
}

\author{
Jim Christenson, Grant Innes, Douglas McKnight, Barb Boychuk, Eric Grafstein, \\ Christopher R. Thompson, Frances Rosenberg, Aslam H. Anis, Ken Gin, Jessica Tilley, \\ Hubert Wong, Joel Singer
}

Abstract

Background: Most Canadian emergency departments use an unstructured, individualized approach to patients with chest pain, without data to support the safety and efficiency of this practice. We sought to determine the proportions of patients with chest discomfort in emergency departments who either had acute coronary syndrome (ACS) and were inappropriately discharged from the emergency department or did not have ACS and were held for investigation.

Methods: Consecutive consenting patients aged 25 years or older presenting with chest discomfort to 2 urban tertiary care emergency departments between June 2000 and April 2001 were prospectively enrolled unless they had a terminal illness, an obvious traumatic cause, a radiographically identifiable cause, severe communication problems or no fixed address in British Columbia or they would not be available for follow-up by telephone. At 30 days we assigned predefined explicit outcome diagnoses: definite ACS (acute myocardial infarction [AMI] or definite unstable angina) or no ACS.

Results: Of 1819 patients, 241 (13.2\%) were assigned a 30-day diagnosis of $\mathrm{AMI}$ and 157 (8.6\%), definite unstable angina. Of these 398 patients, 21 (5.3\%) were discharged from the emergency department without a diagnosis of ACS and without plans for further investigation. The clinical sensitivity for detecting ACS was $94.7 \%$ (95\% confidence interval [Cl] 92.5\%$96.9 \%$ ) and the specificity $73.8 \%(95 \% \mathrm{Cl} 71.5 \%-76.0 \%)$. Of the patients without ACS or an adverse event, $71.1 \%$ were admitted to hospital or held in the emergency department for more than 3 hours.

Interpretation: The current individualized approach to evaluation and disposition of patients with chest discomfort in 2 Canadian tertiary care emergency departments misses 5.3\% of cases of ACS while consuming considerable health care resources for patients without coronary disease. Opportunities exist to improve both safety and efficiency.

CMAJ 2004;170(12):1803-7 very year approximately 62000 Canadians are admitted to hospital with acute myocardial infarction (AMI). ${ }^{1}$ Probably an equal number are admitted with unstable angina. An estimated 300000 to 500000 present to Canadian emergency departments with chest pain; Canadian registry (FASTRAK II) and clinical trial ${ }^{2}$ data suggest that most are admitted to hospital or held for long periods in the emergency department so that AMI can be ruled out. However, some are discharged inappropriately, and "missed MI" remains a serious clinical and medicolegal concern. ${ }^{3-7}$ US studies have revealed that approximately $2 \%$ of patients with AMI or unstable angina are discharged inappropriately. ${ }^{5,8}$

Several risk stratification tools have been developed for use in patients with chest pain and acute coronary syndrome (ACS), ${ }^{910-17}$ but none helps clinicians determine which patients can safely be discharged from the emergency department after a brief assessment. Despite the important and controversial nature of this problem, no Canadian data describe disposition and clinical outcomes of emergency department patients with chest discomfort.

Our objective was to determine the proportion of patients with ACS who are inappropriately discharged from the emergency department and to estimate the hospital stay of patients without ACS. We hypothesized that more than $2 \%$ of patients with ACS would be discharged without ACS being suspected.

\section{Methods}

This prospective, observational cohort study was conducted from June 2000 to April 2001 at St. Paul's and Vancouver General hospitals, which are urban cardiac referral centres. Consecutive patients aged 25 years or older who presented to the emergency departments with chest discomfort were eligible. We excluded those with a clear traumatic cause, a cause that was evident on initial radiographs, enrolment in this study in the previous 30 days, a terminal noncardiac illness with life expectancy of less than 1 year, severe communication problems, no fixed address in British Columbia or no available telephone number for followup. Clinical practice was not affected by the study, and no testing was mandated by protocol.

During daytime hours, research nurses obtained informed consent and enrolled eligible patients. Every morning, eligible patients who had presented during the night were phoned and asked for consent. Information collected for the index visit included time of pain onset, admission and discharge times, initial vital signs, risk factors, disposition, length of hospital stay, results of cardiac consultation, discharge diagnoses, cardiac medications, electrocardiogram (ECG) features, results of tests for cardiac serum markers and other cardiac investigations, and details of adverse events.

Follow-up telephone calls after 30 days included a structured interview to document all health care visits and diagnostic test- 
ing within 30 days after the index presentation. Information was collected on physician and hospital visits and diagnoses, cardiac investigations, adverse events and cardiac medications. For patients lost to phone follow-up, the research nurses contacted local hospital health records departments and searched BC vital statistics databases to identify all hospital visits, diagnoses, procedures and deaths.

After reviewing all information available for the 30 days after presentation, we assigned an outcome diagnosis of AMI, definite unstable angina, possible unstable angina or no ACS. The diagnoses were hierarchic, mutually exclusive and predefined with the use of explicit criteria (Box 1). If such a diagnosis could not be assigned, or if the only criterion was an elevated serum troponin level, 2 cardiologist co-investigators, blinded to each other's assessment, reviewed all the clinical data and assigned an adjudicated outcome diagnosis. The final outcome was determined by agreement of any 2 of the adjudicators and the primary investigator. If all 3 disagreed, the most significant diagnosis was assigned. Cases of AMI and definite unstable angina were classified as definite ACS.

Adverse events defined explicitly before data collection included death, tachycardia, bradycardia or hypotension requiring intervention, proven pulmonary thromboembolism, proven aortic

Box 1: Explicit criteria for outcome diagnoses by 30 days after emergency department presentation with chest discomfort

Acute myocardial infarction (AMI): at least 1 of the following criteria

- Increase in serum creatine kinase MB definite for AMI according to specific hospital criteria or troponin I level $\geq 1.0 \mu \mathrm{g} / \mathrm{L}$

- Diagnostic increase in serum troponin I level $(>0.1$ but $<1.0 \mu \mathrm{g} / \mathrm{L}$ ) and changes consistent with ischemia demonstrated by dynamic electrocardiogram (ECG), > 70\% lesion demonstrated by coronary angiography, positive results of stress test (by radionuclide scan, echocardiography or ECG) or urgent need for revascularization

- ECG evolution consistent with AMI

- Fibrinolytic therapy or primary angioplasty and a clinical diagnosis of AMI

- Death with no other definite cause found

Definite unstable angina: rest pain for $\geq 20 \mathrm{~min}$ and at least 1 of the following criteria

- Increase in serum troponin I level of 0.1 to $0.99 \mu \mathrm{g} / \mathrm{L}$ alone (all adjudicated)

- Dynamic ECG changes consistent with ischemia in 2 contiguous leads (dynamic ST-segment depression $>0.5 \mathrm{~mm}$ or dynamic deep T-wave inversion) but no persistent ST-segment elevation

- $>70 \%$ lesion demonstrated by coronary angiography and hospital admission for acute coronary syndrome (ACS)

- Positive results of stress test (by radionuclide scan, echocardiography or ECG)

Possible unstable angina: rest pain for $\geq 20 \mathrm{~min}$ and a firm clinical diagnosis of unstable angina, with treatment for unstable angina; however, the case did not meet the above criteria for AMI or definite unstable angina

No ACS: applied when the case did not meet the criteria for the other 3 diagnoses aneurysm or dissection, new congestive heart failure requiring intravenous therapy, and instances of either assisted ventilation or chest compressions.

Descriptive statistics, including proportions, medians, means and standard deviations, are reported. Diagnostic sensitivity and specificity, with $95 \%$ confidence intervals (CIs), were calculated with the standard formula for a proportion to classify patients as having definite ACS or not.

This study was approved by the University of British Columbia/Providence Health Care Research Ethics Board.

\section{Results}

Of the 4376 patients screened, 1907 were enrolled. However, 40 (2.1\%) were excluded after enrolment, and $48(2.5 \%)$ had incomplete follow-up. Thus, data for 1819 patients were analyzed. Table 1 summarizes baseline characteristics for the 1819 patients, of whom 241 (13.2\%) had $\mathrm{AMI}$ and 157 (8.6\%) definite unstable angina. Only 18 patients required adjudication of the outcome diagnosis. The 30 -day mortality rate was $1.0 \%$ overall, $5.9 \%$ among patients with AMI and $0.7 \%$ among those with definite unstable angina.

Fig. 1 relates patient outcome to emergency department disposition. Of the 660 patients admitted to hospital, 244 (37.0\%) did not have ACS or an adverse event. Of the 1334 patients without ACS or an adverse event, 948 (71.1\%)

\section{Table 1: Baseline characteristics for 1819 consecutive,} consenting patients

\begin{tabular}{lr}
\hline Previous conditions, no. (and \%) of patients & \\
AMI & $389(22.0)$ \\
Angina & $618(35.1)$ \\
Cocaine use & $28(1.6)$ \\
Logistics & $563(31.0)$ \\
Arrived by ambulance, no. (and \%) of patients & \\
Median time, minutes (25th, 75th percentiles) & $125(61,316)$ \\
$\quad$ From pain onset to arrival & $31(19,56)$ \\
From arrival to first electrocardiogram & \\
Demographic characteristics & $58.2(16.1)$ \\
Age, mean (and SD), yr & $1051(57.8)$ \\
Male, no. (and \%) of patients & \\
Vital signs, mean (and SD) & \\
Blood pressure, mm Hg & $144.1(27.1)$ \\
Systolic & $81.5(15.4)$ \\
Diastolic & $80.9(20.8)$ \\
Heart rate, beats/min & $18.8 \quad(3.5)$ \\
Respiratory rate, breaths/min & \\
30-day diagnosis, no. (and \%) of patients & $241(13.2)$ \\
Definite AMI & $157 \quad(8.6)$ \\
Definite unstable angina & $50(2.7)$ \\
Possible unstable angina & $83(4.6)$ \\
Adverse event but no ACS & $1288(70.8)$ \\
No ACS or adverse event & \\
\hline
\end{tabular}

Note: Percentages are based on the number of patients with confirmed, accurate data. $\mathrm{SD}=$ standard deviation, $\mathrm{AMI}=$ acute myocardial infarction, $\mathrm{ACS}=$ acute coronary syndrome. 
were admitted or stayed longer than 3 hours in the emergency department.

At the end of the evaluation in the emergency department, ACS was suspected in 750 cases and unsuspected in 1069 (Table 2). Of the 398 patients with a 30-day diagnosis of definite ACS, 21 (5.3\%) were discharged without suspicion of the disease; thus, the clinical sensitivity for ACS was $94.7 \%$. These 21 patients included 11 (4.6\%) of the 241 with $\mathrm{AMI}$ and $10(6.4 \%)$ of the 157 with definite unstable angina. One of the 21 patients died during the 30-day follow-up period. The true diagnosis was made from 8 hours to 28 days after the index presentation and most commonly during a return visit because of recurrent symptoms. Only 7 of the 21 patients had negative results of serum marker tests and a normal ECG during the index presentation. The other 14 had low-level serum marker elevations or high-risk ECG features. The most common discharge diagnoses at the index presentation of these 21 patients were chest pain not yet determined ${ }^{5}$ and atypical chest pain. ${ }^{3}$

Percutaneous intervention was performed in $48.1 \%$ of the patients with AMI and $33.8 \%$ of the patients with unstable angina. The rates of coronary artery bypass grafting (CABG) were $10.4 \%$ and $19.7 \%$ respectively. Pulmonary embolism was confirmed in 4 patients, 1 of whom died in hospital; no cases were missed at the index presentation. Aortic aneurysm or dissection was confirmed in no patients.

\section{Interpretation}

In this study, 398 of 1819 patients evaluated for chest discomfort had a diagnosis of ACS confirmed within 30 days; $21(5.3 \%)$ of the 398 had been discharged from the emergency department without suspicion of ACS. This "miss rate" is more than twice that reported by Pope and colleagues $^{8}$ from a large US study $(2.1 \%)$. In a previous survey of Canadian emergency physicians, ${ }^{18}$ only $5 \%$ reported using a systematic follow-up process to identify missed cases, but half estimated that their miss rate for AMI was greater than 2\%. Most (94\%) indicated that an earlydischarge prediction tool would be helpful as long as it did not increase the rate of missed AMI above 2\%. Canadian emergency physicians would probably consider the miss rate of $5.3 \%$ for ACS in this study unacceptable.

Many emergency departments in the United States have developed chest pain evaluation units (CPEUs) to reduce the likelihood of discharge of patients with ACS. For 6 to

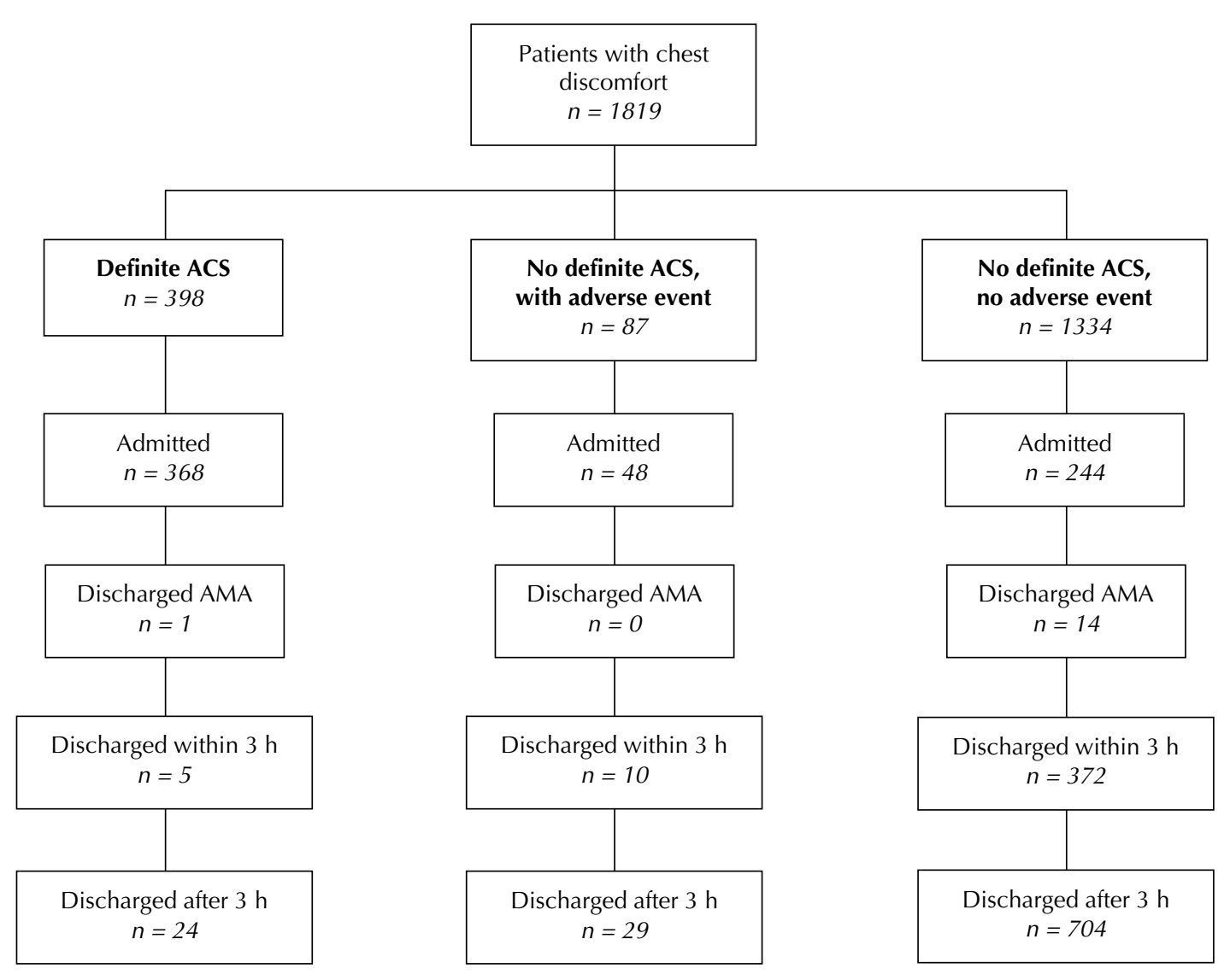

Fig. 1: Disposition and 30-day outcomes of consecutive consenting patients aged 25 years or older presenting with chest discomfort to 2 urban tertiary care emergency departments. ACS = acute coronary syndrome, AMA = against medical advice. 
12 hours, these CPEUs apply intensive diagnostic pathways that incorporate continuous monitoring, serial ECGs, serial marker assays, stress tests and advanced imaging for patients with a low prevalence of ACS $(2 \%-5 \%) .^{19-27}$ This approach may improve diagnostic safety, but it increases costs and has not been widely embraced in Canada. CPEUs are cost-effective relative to admitting all low-risk patients to coronary care units ${ }^{21,24,28-32}$ but have never been compared with the unstructured diagnostic approach used in most Canadian hospitals. Existing diagnostic pathways and guidelines $^{10,13,16,17,33-38}$ do not include clear guidance for the early discharge of patients with a very low likelihood of disease. To ensure that we miss less than $2 \%$ of patients, clinicians need effective tools and diagnostic pathways. To maximize efficiency and preserve limited health care resources, administrators and clinicians need evidence that new models are more cost-effective than current Canadian practice.

This study has provided the most current and accurate information on ACS diagnosis in Canadian emergency departments. We attempted to enrol consecutive consenting patients with a presenting symptom of chest discomfort; hence, our study sample is more representative of patients arriving with chest pain than are samples in randomized, interventional clinical trials. Our findings are more robust than registry data because we developed explicit definitions a priori and used structured data-collection and follow-up mechanisms. Our 97\% direct follow-up rate is excellent, and at 30 days only $2.7 \%$ of the patients remained in the ill-defined diagnostic category of possible unstable angina.

There are, however, important study limitations. Some very sick patients who could not provide informed consent were excluded. This might account for the relatively low $30-$ day mortality rate in our AMI population and would also increase our apparent miss rate. It is possible that the standard of practice improved during the study, since clinicians were aware of outcome monitoring. We based inclusion on the presence of chest discomfort and therefore, by design, did not enrol ACS patients who had no pain. Although this is a limitation, it was necessary so that we could focus on a definable patient population. We did not mandate follow-up marker tests and ECGs for all patients. Therefore, it is possible that some AMIs were undetected; but, if so, there were no apparent sequelae by 30 days. Mandating a structured set of diagnostic tests for all patients would have increased study costs prohibitively for a small and, arguably, unnecessary gain in diagnostic confidence. We relied on patient contact at about 30 days to determine outcome but did not rely on the patient's interpretation of events. The patient informed research assistants of hospital and physician visits and all diagnostic testing. We contacted the physicians to confirm the diagnostic impression and reviewed all admission documentation and diagnostic reports to accurately determine the explicitly defined final outcome.

Many ACS studies have combined "softer" outcomes, such as percutaneous intervention or CABG, or readmission because of unstable angina, in a composite outcome. Since some patients undergo elective percutaneous intervention or CABG during the 30 days after initial presentation with chest discomfort, we tried to determine outcome independent of these events using a priori definitions and an adjudication panel when necessary.

Information from other Canadian settings is needed to clarify misdiagnosis rates and utilization of hospital resources across the country.

\section{Conclusion}

The current individualized approach to evaluation and disposition of cases of chest discomfort in 2 Canadian emergency departments misses $5.3 \%$ of cases of ACS while consuming considerable health care resources in dealing with most of the patients without ACS. Opportunities exist to improve both safety and efficiency. Clinical tools are needed to help clinicians identify patients who can safely be discharged after a short period of investigation.

This article has been peer reviewed.

From the Departments of Medicine (Thompson, Gin), Surgery (Christenson, Innes, McKnight, Grafstein), Pathology and Laboratory Medicine (Rosenberg), and Health Care and Epidemiology (Anis, Wong, Singer), University of British Columbia, the Centre for Health Evaluation and Outcome Sciences (Christenson, Innes, Grafstein, Anis, Tilley), the Departments of Emergency Medicine (Christenson, Innes, Boychuk, Grafstein), Medicine (Thompson), and Pathology and Laboratory Medicine (Rosenberg), Providence Health Care, St. Paul's Hospital, and the Departments of Emergency Medicine (McKnight) and Medicine (Gin), Vancouver Hospital, Vancouver, BC

Table 2: Accuracy of diagnostic suspicion about ACS at index presentation

\begin{tabular}{|c|c|c|c|}
\hline \multirow[b]{2}{*}{ Suspected diagnosis } & \multicolumn{2}{|c|}{ 30-day diagnosis; no. of patients } & \multirow[b]{2}{*}{$\begin{array}{l}\text { Total no. } \\
\text { of patients }\end{array}$} \\
\hline & $\begin{array}{l}\text { AMI or definite } \\
\text { unstable angina }\end{array}$ & $\begin{array}{c}\text { No AMI or definite } \\
\text { unstable angina }\end{array}$ & \\
\hline ACS* & 377 & 373 & 750 \\
\hline No ACS† & 21 & 1048 & 1069 \\
\hline Total no. of patients & 398 & 1421 & 1819 \\
\hline \multicolumn{4}{|c|}{$\begin{array}{l}\text { *No. of patients admitted or discharged with a diagnosis of definite or possible ACS. } \\
\text { †No. of patients discharged with a diagnosis of no ACS. } \\
\text { Sensitivity } 94.7 \%(377 / 398), 95 \% \text { confidence interval (Cl) } 92.5 \%-96.9 \% \text {. } \\
\text { Specificity } 73.8 \%(1048 / 1421), 95 \% \mathrm{Cl} 71.5 \%-76.0 \% \text {. } \\
\text { Positive predictive value } 50.3 \%(377 / 750), 95 \% \mathrm{Cl} 46.7 \%-53.8 \% \text {. } \\
\text { Negative predictive value } 98.0 \%(1048 / 1069), 95 \% \mathrm{Cl} 97.2 \%-98.9 \% \text {. }\end{array}$} \\
\hline
\end{tabular}




\section{Competing interests: None declared.}

Contributors: Principal investigator Jim Christenson envisioned and designed the trial and was responsible for all aspects; he led the data analysis, results interpretation and manuscript writing. The project's initial instigators, Grant Innes and Eric Grafstein, evaluated resource utilization in the emergency department at St. Paul's Hospital and contributed to analysis and interpretation and to manuscript writing. Douglas McKnight was responsible for data collection at one site and contributed to outcome designations and results interpretation. Barb Boychuk was responsible for patient enrolment, oversaw data collection and processing, and managed the research staff; she wrote portions of the manuscript. Christopher Thompson critically evaluated trial design, methodology and analysis. Frances Rosenberg coordinated the additional laboratory testing and was responsible for its quality; she also aided in interpretation and manuscript writing. Aslam Anis contributed to design of methodology. Jessica Tilley was responsible for data cleaning and analysis. Hubert Wong oversaw the analysis and contributed to writing of the methods. Joe Singer provided critical advice on research design and was responsible for implementation of the database, data entry and data analysis. Christopher Thompson, Aslam Anis, Ken Gin, Jessica Tilley, Hubert Wong and Joel Singer provided critical review of the manuscript for important intellectual content. All authors approved the version to be published.

Acknowledgements: This study was supported by grant 38023 from the Canadian Institutes of Health Research.

\section{References}

1. Heart and Stroke Foundation of Canada. The changing face of heart disease and stroke in Canada. Ottawa: The Foundation; 2000.

2. Gibler WB, Hoekstra JW, Weaver WD, Krucoff MW, Hallstrom AP, Jackson RE, et al. A randomized trial of the effects of early cardiac marker availability on reperfusion therapy in patients with acute myocardial infarction: the Serial Markers in Acute Myocardial Infarction Rapid Treatment Trial. 7 Am Coll Cardiol 2000;36:1500-6.

3. Zarling EJ, Sexton H, Milnor P. Failure to diagnose acute myocardial infarction: the clinicopathologic experience at a large community hospital. $7 A M A$ 1983;250:1177-81.

4. Lee TH, Rouan GW, Weisberg MC, Brand DA, Acampora D, Stasiulewicz $\mathrm{C}$, et al. Clinical characteristics and natural history of patients with acute myocardial infarction sent home from the emergency room. Am 7 Cardiol 1987; 60:219-24

5. McCarthy BD, Beshansky JR, D’Agostino RB, Selker HP. Missed diagnoses of acute myocardial infarction in the emergency department: results from a multicenter study. Ann Emerg Med 1993;22:579-82.

6. Tierney WM, Fitzgerald J, McHenry R, Roth BJ, Psaty B, Stump DL, et al. Physicians' estimates of the probability of myocardial infarction in emergency room patients with chest pain. Med Decis Making 1986;6(1):12-7.

7. Rusnak RA, Stair TO, Hansen K, Fastow JS. Litigation against the emergency physician: common features in the cases of missed myocardial infarction. Ann Emerg Med 1989;18:1029-34.

8. Pope JH, Aufderheide TP, Ruthazer R, Woolard RH, Feldman JA, Beshansky JR, et al. Missed diagnoses of acute cardiac ischemia in the emergency department. NEngl 7 Med 2000;342:1163-70.

9. Antman E, Cohen M, Bernink P, McCabe C, Horacek T, Papuchis G, et al. The TIMI Risk Score for unstable angina/non-ST-elevation MI: a method for prognostication and therapeutic decision making. FAMA 2000;284:835-42.

10. Goldman L, Cook E, Brand D. A computer protocol to predict myocardia infarction in emergency department patients with chest pain. $N$ Engl $7 \mathrm{Med}$ 1988:318:797-803

11. Goldman L, Weinberg M, Weisberg M, Olshen R, Cook EF, Sargent RK, et al. A computer-derived protocol to aid in the diagnosis of emergency room patients with acute chest pain. NEngl 7 Med 1982;307:588-96.

12. Lee T, Rouan G, Weisberg M, Brand D, Cook F, Acampora D, et al. Sensitivity of routine clinical criteria for diagnosing myocardial infarction within 24 hours of hospitalization. Ann Intern Med 1987;106:181-6.

13. Lee TH, Pearson SD, Johnson PA, Garcia TB, Weisberg MC, Guadagnoli E, et al. Failure of information as an intervention to modify clinical management. A time-series trial in patients with acute chest pain. Ann Intern Med 1995;122:434-7.

14. Limkakeng A, Gibler W, Pollack C, Hoekstra J, Sites F, Shofer F, et al. Combination of Goldman risk and initial cardiac troponin I for emergency department chest pain risk stratification. Acad Emerg Med 2001;8:696-702.

15. Selker H, Beshansky J, Griffith J, Aufderheide T, Ballin D, Bernard S, et al. Use of the Acute Cardiac Ischemia Time-Insensitive Predictive Instrument (ACI-TIPI) to assist with triage of patients with chest pain or other symptoms suggestive of acute cardiac ischemia: a multicenter, controlled clinical trial. Ann Intern Med 1998;129:845-55.

16. Fitchett D, Goodman S, Langer A. New advances in the management of acute coronary syndromes. CMA7 2001;164:1309-16.

17. Tatum JL, Jesse RL, Kontos MC, Nicholson CS, Schmidt KL, Roberts CS, et al. Comprehensive strategy for the evaluation and triage of the chest pain patient. Ann Emerg Med 1997;29:116-25.

18. MacGougan C, Christenson J, Innes G, Raboud J. Emergency physicians' attitudes towards a clinical prediction rule for the safe, early discharge of patients with chest discomfort. Can 7 Emerg Med 2000;3:89-94.

19. Costi A, Paladini B, Toccafondi S, Magazzini S, Olivotto I, Galassi F, et al. Effectiveness of a multidisciplinary chest pain unit for the assessment of coronary syndromes and risk stratification in the Florence area. Am Heart 72002 144:630-5

20. Dallara J, Severance HD, Davis B, Schulz G. Differences between chest pain observation service patients and admitted "rule-out myocardial infarction" patients. Acad Emerg Med 1997;4:693-98.

21. DeLeon AC, Farmer CA, King G. Chest pain evaluation unit: a cost-effective approach for ruling out myocardial infarction. South Med J 1989;82:1083-89.

22. Farkouh M, Smars P, Reeder G, Zinsmeister A, Evans R, Meloy T, et al. A clinical trial of a chest-pain observation unit for patients with unstable angina. N Engl 7 Med 1998;339:1882-8.

23. Gaspoz J, Lee T, Cook F, Weisberg M, Goldman L. Outcome of patients who were admitted to a new short-stay unit to "rule-out" myocardial infarction. Am 7 Cardiol 1991;68:145-9.

24. Gaspoz JM, Lee TH, Weinstein MC, Cook EF, Goldman P, Komaroff AL, et al. Cost-effectiveness of a new short-stay unit to "rule out" acute myocardial infarction in low risk patients. 7 Am Coll Cardiol 1994;24:1249-59.

25. Gibler WB, Runyon JP, Levy RC, Sayre MR, Kacich R, Hattemer CR, et al A rapid diagnostic and treatment center for patients with chest pain in the emergency department. Ann Emerg Med 1995;25:1-8.

26. Stomel R, Grant R, Eagle KA. Lessons learned from a community hospital chest pain center. Am 7 Cardiol 1999;83:1033-7.

27. Zalenski RJ, Rydman RJ. Prevalence, characteristics, and organizational context of emergency department chest pain centers in the United States. Circulation 1996;94(8):I-50.

28. Bahr RD. Chest pain centers: moving toward proactive acute coronary care. Int 7 Cardiol 2000;72:101-10.

29. McCullough PA, Ayad O, O'Neill WW, Goldstein JA Costs and outcomes of patients admitted with chest pain and essentially normal electrocardiograms. Clin Cardiol 1998;21:22-6.

30. Mikhail MG, Smith FA, Gray M, Britton C, Frederiksen SM. Cost-effectiveness of mandatory stress testing in chest pain center patients. Ann Emerg Med 1997;29:88-98

31. Tosteson AN, Goldman L, Udvarhelyi IS, Lee TH. Cost-effectiveness of a coronary care unit versus an intermediate care unit for emergency department patients with chest pain. Circulation 1996;94:143-50.

32. Gomez MA, Anderson JL, Karagounis LA, Muhlestein JB, Mooers FB. An emergency department-based protocol for rapidly ruling out myocardial ischemia reduces hospital time and expense: results of a randomized study (ROMIO). 7 Am Coll Cardiol 1996;28:25-33.

33. Braunwald E, Antman EM, Beasley JW, Califf RM, Cheitlin MD, Hochman JS, et al; Committee on the Management of Patients with Unstable Angina ACC/AHA 2002 guideline update for the management of patients with unstable angina and non-ST-segment elevation myocardial infarction: a report of the American College of Cardiology/American Heart Association Task Force on Practice Guidelines. 2002. Available: www.acc.org/clinical/guidelines/unstable /incorporated/index.htm (accessed 2004 Mar 18)

34. Braunwald E, Antman EM, Beasley JW, Califf RM, Cheitlin MD, Hochman JS, et al; Committee on the Management of Patients with Unstable Angina. ACC/AHA 2002 guideline update for the management of patients with unstable angina and non-ST-segment elevation myocardial infarction - summary article: a report of the American College of Cardiology/American Heart Association Task Force on Practice Guidelines. 7 Am Coll Cardiol 2002;40:1366-74.

35. Bertrand M, Simoons ML, Fox K, Wallentin L, Hamm C, McFadden E, et al. Management of acute coronary syndromes in patients presenting without persistent ST-elevation. Eur Heart 7 2002;23:1809-40.

36. Nichol G, Walls R, Goldman L, Pearson S, Hartley LH, Antman E, et al. A critical pathway for management of patients with acute chest pain who are at low risk for myocardial ischemia: recommendations and potential impact. Ann Intern Med 1997;127:996-1005.

37. Selker H, D'Agostino R, Laks M. A predictive instrument for acute ischemic heart disease to improve coronary care unit admission practices: a potentia on-line tool in a computerized electrocardiograph. F Electrocardiol 1988;21 (Suppl):S11-S7.

38. Hamm CW, Goldmann BU, Heeschen C, Kreymann G, Berger J, Meinertz $\mathrm{T}$. Emergency room triage of patients with acute chest pain by means of rapid testing for cardiac troponin T or troponin I. N Engl 7 Med 1997;337:1648-53.

Correspondence to: Dr. Jim Christenson, Department of Emergency Medicine, St. Paul's Hospital, 1081 Burrard St., Vancouver BC V6Z 1 Y6; fax 604 806-8798;

jimchris@interchange.ubc.ca 